\title{
Exploring the Affordances and Musico-Spatial Performance Opportunities of a Virtual Drumming Environment
}

\author{
Jeremy Ham ${ }^{1}$, Uwe Woessner ${ }^{2}$, Joachim Kieferle ${ }^{3}$, Lawrence Harvey ${ }^{4}$ \\ ${ }^{1}$ FUNKarchitects ${ }^{2}$ University of Stuttgart HLRS ${ }^{3}$ Hochschule Rheinmain ${ }^{4}$ RMIT \\ University \\ Ijeremy@funkarchitects.net ${ }^{2}$ woessner@hlrs.de ${ }^{3}$ joachim.kieferle@hs-rm. \\ de Lawrence.Harvey@rmit.edu.au
}

The intersection of music and architecture (spatial design) has remained a fascination for practitioners and researchers for many years. This paper reports on the development of a Virtual Drumming Environment (VDE) that provides a research and cross-domain performance environment for musico-spatial design research explorations. The VDE is explored as a means of revealing affordances related to the complexities of polyrhythmic drumming through cross-domain representation of MIDI data as polyrhythmic event-time-dynamics molecules (PM) within virtual space. Further to this, we explore the VDE as a cross-domain performance environment where new modes of musico-spatial improvisation are revealed that extend drumming's 'known worlds' (Bruford, 2015).

Keywords: Music and Architecture, Cross-Domain Representation, Virtual Reality, Musico-Spatial performance, Affordance

\section{INTRODUCTION}

This paper outlines a Virtual Drumming Environment (VDE) that bridges the nexus between music and spatial design through live performance. The VDE is a design research tool that integrates the digital drum kit, Virtual Reality (VR) and Virtual Instrumentation (VI). The VDE provides a platform for cross-domain performance within the musical, spatial and 'musicospatial' contexts. In addition, the VDE offers opportunities to discover new insights into the complexities of polyrhythmic drumming through Cross-Domain Representation (XDR) of music in the spatial domain.

Many practitioners, researchers and creatives have explored the intersection of the musical and spatial domains (as music and architecture) from various philosophical, historical and theoretical, technological and scientific perspectives. We are interested in the transformation of musical data into the spatial domain as a means of learning more about music (and in this case, polyrhythmic drumming). We are also interested in using improvised live performance on the digital drum kit as a driver for cross-domain musico-spatial design research explorations.

We propose that cross-domain design research can enable reflective insights into the spectrum of knowledge within the musical and spatial domains (Figure 1). In this way, the spatialisation of music as 'frozen music' (Eckerman, 1829), 'archimusic' (No- 
vak, 1994) and the 'Y-Condition' (Martin, 1994) serves both as an extension of the cross-domain creative processes so brilliantly undertaken by lannis Xenakis, and as a vehicle for unlocking new knowledge in both domains.

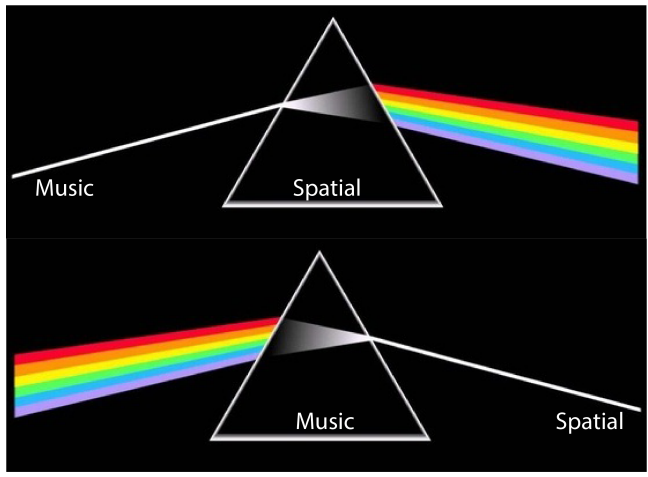

\section{A VIRTUAL DRUMMING ENVIRONMENT (VDE)}

The VDE is a custom-designed virtual drumming environment designed as a platform to enable dualskilled musician-spatial designers to generate both musical and spatial output through playing the drums. The VDE is based on a purpose-driven application within the Covise and OpenCover Virtual Reality (VR) visualisation suite developed at the University of Stuttgart High Performance Computing Centre (HLRS). VR spatialisation is generated in real time by the translation of MIDI signals (time, note events and note velocity, described as dynamic note events-intime) from a Roland TD20 digital drum kit into spatial parameters. VR output is experienced through the interface of a HTC Vive head-mounted display (HMD), inside a CAVE and as screen-based projections. The workflow for the VDE is described in Figure 2.

The principle idea behind the VDE is to push the boundaries of the digital drum kit as a tool for sound synthesis and spatialisation. The sound output from the VDE works across the range of modelled drum kit sounds from the TD20 to synthesised Vir- tual Instrument (VI) sounds from the Spectrasonics Omnisphere Kontakt plugin within the Reaper digital audio workstation. Thus, a single strike on a drum pad can be transformed from the familiar crack of a snare drum to a vast soundscape of sounds accompanied by real-time spatial projections in virtual reality (VI+VR). This comprehensive musico-spatial work environment provides a first-person (drummer-centric) real-time spatialisation and musical expression environment through which to experiment with the musical and spatial aspects of dynamic polyrhythmic drumming.

The design research question inherent in this project is: how does one represent the output of the digital drum kit within the spatial domain? The VDE adopts a spatial schema for the VR spatialisation based on ideas of a 3D Spatial Drum Notation (3DSDN) (see Ham, 2017). The 3D-SDN utilises the threedimensionality of CAD-based and virtual environments to represent the spatiality of the drum kit as a means of addressing the shortcomings of traditional stave-based drum notation. The core of the VDE is a modelled representation of the digital drum kit and a radial note schema, from which MIDI drum notes are projected into virtual space. The modelled representation of the drum kit was derived from a scan of the real kit to build a spatially accurate 3D Studio Max model of the digital drum kit that is exported as VRML for use in Open Cover. This virtual model matches the physical drum kit exactly, thus allowing the drummer to accurately play the kit even with the HMD on.

This VRML model also contains the piped spatial schema for note setout and a coordinate system to locate the virtual drum kit inside the VR environment (see Figure 3). This model has its origin set to a point on the TD20 drum rack and, using the 'calibrate to hand' function of the Tablet UI, the model can be accurately positioned within the virtual environment. Drum note generation points are distributed radially around a circular schema set to the origin on the virtual drum kit model. In this schema, the vector, velocity and radius of spherical drum notes are generated as functions of velocity MIDI data (between 0
Figure 1

Cross-domain design research as a 'reflective lens'. 
Figure 2

VDE workflow.

Figure 3

The VDE VRML template.

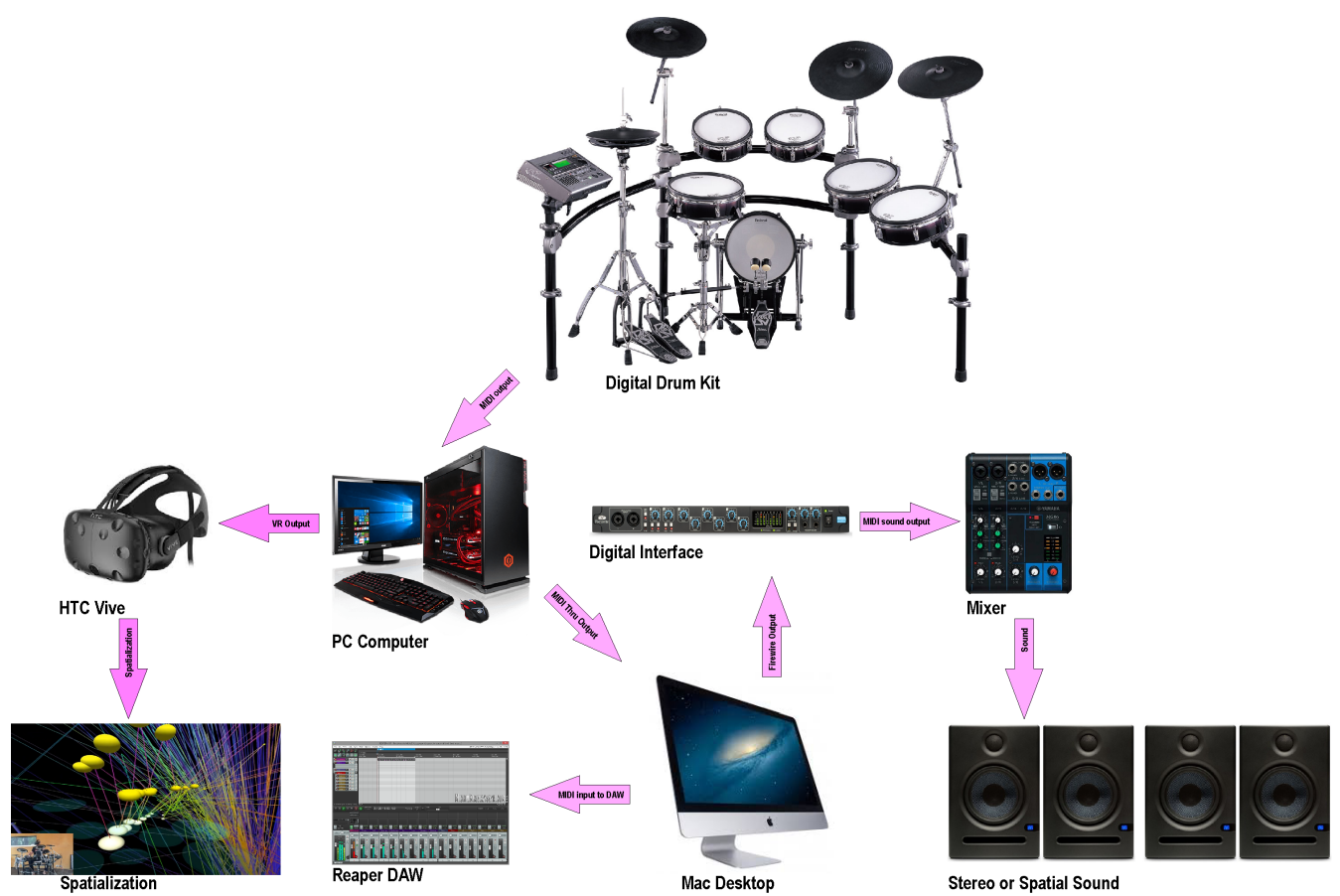

and 127). Velocities are set out along a arced trajectory axis so that low-velocity playing projects smalldiameter spherical notes towards the drummer and high-velocity playing projects larger diameter notes away from the drummer. These spherical notes are connected by pipes lines between note centrepoints.

We describe these spatial artefacts as 'Polyrhythmic Event-Time-Dynamics Molecules' (PM). PM transform the MIDI parameters of drum events in time (what notes are played, and when) and dynamics (velocity) into variably-sized polyrhythmic molecules that act as a form of real-time drum notation and spatialisation. The trajectory of these notes is overtaken by the VR physics engine, projecting notes into the distance. The result, when viewed in the HTC Vive, is a spectacular dynamic spatialisation of polyrhythmic drumming as a web of historic PM rotating around the user in virtual space.

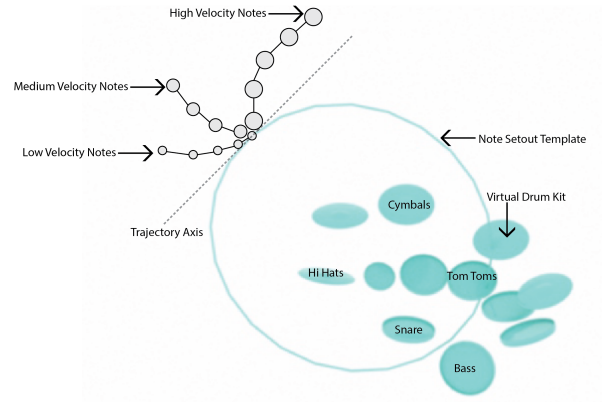




\section{AFFORDANCES OF THE VDE}

The next design research question relates to how the VR environment, dynamic spatial output and the spatial schema provide opportunities for deepening understandings of polyrhythmic drumming. That is; how does the VDE provide affordances for understanding music? The concept of affordance has its origins in the field of evolutionary psychology and was related to an animal's relationship with the environment (Gibson, 1979). Donald Norman in The Design of Everyday Things introduces the idea of affordance (Norman, 2002) relating Gibson's ideas to the field of design. In this domain, an 'affordance is what one system (say, an artefact) provides to another system (say, a user). The concept of affordance is relational because of the complementarity entailed between two interacting systems' (Maier and Fadel, 2009). The central idea is that representations and objects provide an affordance by providing an indication of their use or meaning. Affordances can be enhanced through engagement with appropriate metaphors and by following a coherent conceptual model in interface design.

The potential affordances of the VDE are summarised in relation to this research:

- The output of MIDI data in VR as dynamic virtual objects.

- The output of MIDI data as sound, connected in real time to dynamic spatial output in VR.

- Real-time processing and output of MIDI data from the digital drum kit.

- Navigation and immersion of MIDI output as $3 \mathrm{D}$ virtual objects.

- Use of geometry and colour in spatial output.

- The ability to create virtual galleries of static polyrhythmic molecules for comparison and review.

We conceptualise dynamic polyrhythmic drumming as a creative activity akin to the design process- as a design-like process that involves the designerly placement of dynamic note events-in-time using hands and feet on the interface of the digital drum kit. The VDE, as a real-time VR-based spatialisation of these musical design decisions, provides a dynamic spatial definition that engages a different set of affordances that are otherwise unavailable in the sonic domain. Whilst traditional stave-based notation may be useful as a basic representation of simple drumming, these do not adequately communicate some of the complexities of difficult-to-play drumming.

In particular, the connections between drumming as dynamic note events in time provide insights into hand-foot drumming combinations used in beat making and drum solos. Taking an example of a typical Snare - Bass Drum triplet pattern, these notes are spatialised as a triangulation defined by three spheres appearing before the eyes and projecting forward in virtual space. When the triplet is played harder (higher note velocity), slower or faster, the triangulation, sphere diameter and note trajectory change accordingly (Figure 4).

When playing within the VDE, connections can be made in the mind between the real-time sonic and spatial outputs. Regularity, repetition and exact timing are rewarded by an ordered spatial output and a well-played, perfectly timed beat looks as good as it sounds. Complex, polyrhythmic drumming departs from the regularity of beat-making to be defined by dense entanglements of PM in virtual space. The great differences between, for example, the hyper-complex time signatures, syncopation and polyrhythmic drumming of The Mars Volta and the ordered straight time 4:4 beats of the Beatles, are evident when played in the VDE. Drumming techniques regularly used in beat making and soloing such as triplets, flams and 4th, 8th and 16th note rolls are given an unique spatial identity. These spatial outputs can be learnt so that the drummer becomes familiar with musico-spatial output. This important first stage of learning the system informs the next stage of working with the VDE.

The Covise code for the VDE provides for drumming patterns to be sampled and collated to form scaled static PM virtual galleries (Figure 5) by striking a dedicated drum pad. This works by first playing a 
Figure 4

VDE view from

drummers

perspective

showing PM traces

(main image) and

view of drummer

(inset image)

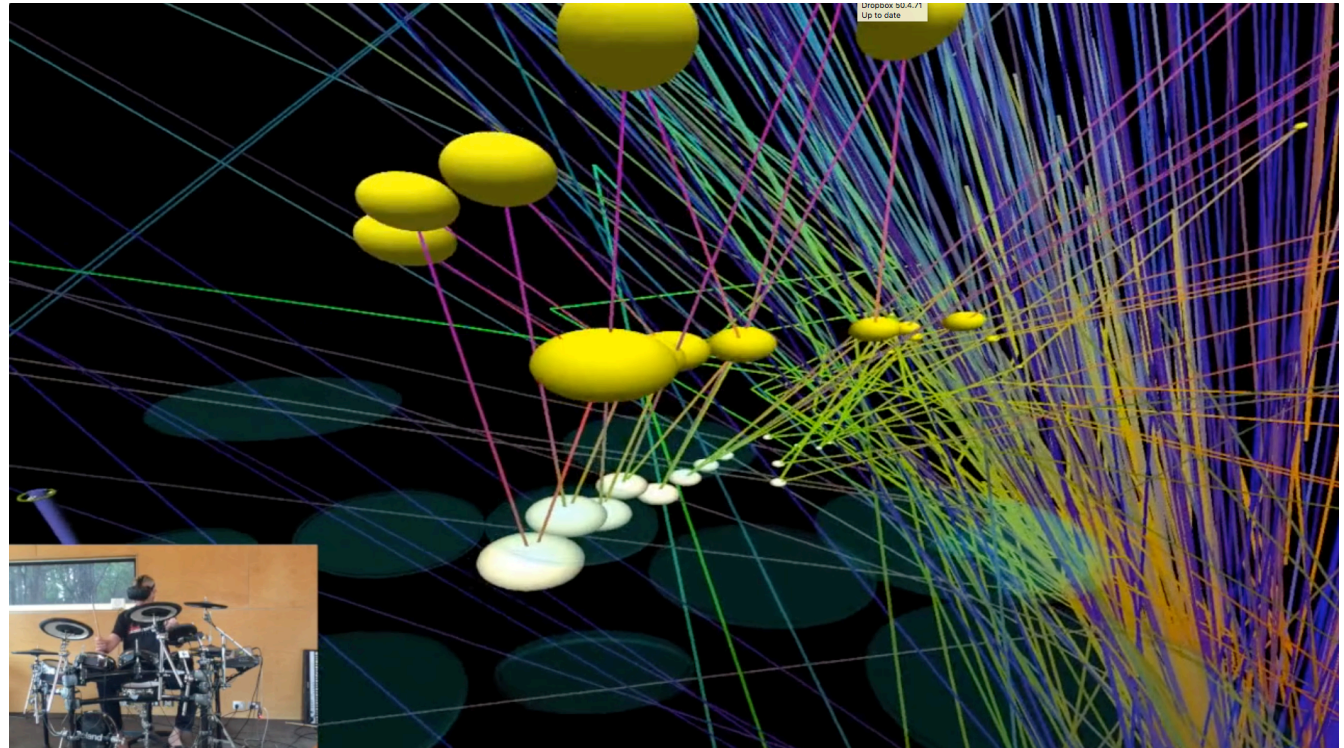

pattern on the digital drum kit then striking the sampling pad resulting in the storing, scaling down and placement of the PM for that pattern in a set grid in a Virtual Gallery. These scaled frozen representations of PM can be reviewed by picking up, zooming, rotating and review using the HTC Vive head and hand gear. These PM can also be exported to 3D Studio Max and other applications to form the basis of further transformations and explorations in fabrication and 3D printing.

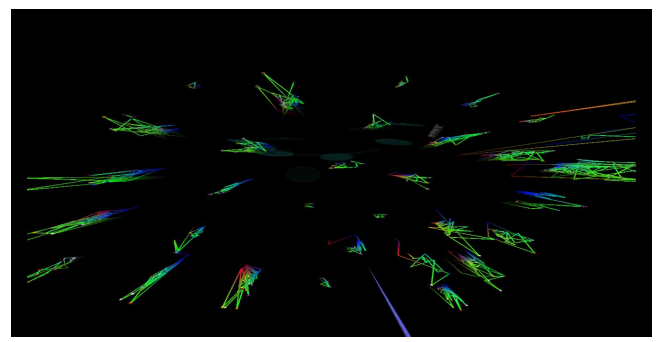

This capacity brings forward issues relating to the the comparable affordances of static versus dynamic XDR of polyrhythmic drumming. Dynamic VDE PM allow real-time testing, experimentation and analysis of drumming where actions, sounds and spatialisations are connected in time. In comparison, static PM in virtual galleries comprise collections of dynamic note events frozen in time. The differences between the affordances of the dynamic, and those of the static are complimentary and, together, they offer several pathways through which to interrogate the complexities of drumming.

The VDE can be used within a tutoring environment to assist students explore drumming. Recognising that students engage in learning through styles that include visual, auditory and kinaesthetic, the real-time connection between drumming performance and sound output and spatialisation in the VDE provides unique opportunities to enhance the learning of drums through engagement in the visual and spatial domains. The game-like novelty of this VR 
environment and its affordances may help to inform children on aspects of timing, pattern-making, performance consistency and dynamics as a real-time 3D spatial notation system (3D-SDN) (see Ham, 2017 for previous work on the 3D-SDN).

This spectacular visual environment may also offer potential as a synaesthetic interface for the description of drumming, rhythm, beat-making and other aspects of music. This may be especially instructive for hearing-impaired persons where the qualities of polyrhythmic drumming can be ekphrastically described as dynamic spatial PM in a virtual environment. This could be used, in association with the sensation of air pressure output from sub-woofer speakers, as a means of imparting some of the feeling and enjoyment of musical performance to persons with different sensory capacities.

This first stage of working with the VDE operated from an analytical design research modality. Digital drumming was provided a virtual spatial definition that represented the complexities of polyrhythmic playing. This first analytical stage has been complimented by creative musico-spatial explorations that have been based on learning the system and applying this learning to a live performance environment.

\section{THE VDE AS A MUSICO-SPATIAL PERFOR- MANCE ENVIRONMENT}

To achieve the integrated Virtual Instrumentation and Virtual Reality (VI+VR) VDE, the MIDI out from the digital drum kit is split; with one cable directed to the VR PC and the second directed to the digital interface for input to the Reaper DAW. Using the Spectrasonics Omnisphere synthesiser package, 12,000 sounds are enabled along with granular and wavetable synthesis and special effects capabilities. The VI+VR VDE works as a rich environment for drumming performance where new potentials are informed by a spectacular sonic and virtual environment. This VDE thus allows us to extend 'drumming's known world' through drumming performance that is, by its very nature, situated at the compositional end of what Bill Bruford describes as the Functional-Compositional
Continuum (FCC) (Bruford, 2015) . In doing this, we work outside of our known boundaries of musical and spatial practice to forge new territories of musico-spatial creative practice.

The wider research project (see Ham, 2017, Ham 2017a, Ham 2018 and Ham, 2018a) has used improvisation as a generative methodology for design research. Improvising, experimenting and testing the VDE revealed multiple musico-spatial improvisational performance modalities:

- playing with the sound output from the digital drum kit (musical improvisation);

- playing with the generation of spatial output in an immersive VR environment (spatial improvisation);

- playing with both sound output and spatial output at the same time (musico-spatial improvisation);

- playing with both sound output and spatial output at the same time, with synthesised sounds derived from virtual instrumentation (augmented musico-spatial improvisation);

This new form of musico-spatial improvisation - improvisation in both the musical and spatial domains within the same performance- engages the crossdomain practitioner's capacities as drummer and spatial designer. Learned expertise in drumming as the designerly actuation of dynamic musical events-intime is redirected towards musico-spatial design in both time and space. This virtual output provides a series of augmented musico-spatial feedback loops, where initial improvised musical intention generates a dynamic VR artefacts that pique the drummer's interest which are responded to by generating secondorder musico-spatial output. This cyclical secondorder improvisation then generates further rounds of sonic and spatial outputs inside the VDE. 
Figure 6

Performance setup with digital drum kit and screen projection of HMD view of PM Virtual Gallery.

Figure 7

Performing with the VDE.

Figure 8

Playing the Sound Cave VDE inside the HLRS CAVE.
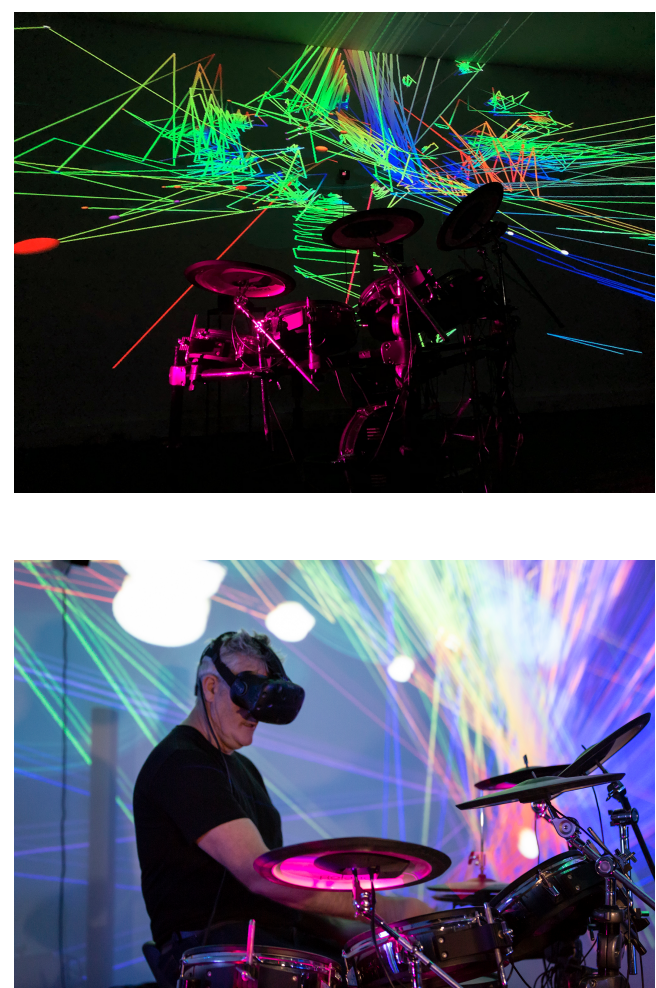

\section{Extending the VDE}

Collaborative development between the authors in Australia and Germany has extended the VDE on two fronts. The first extension involves the development of the Covise code to allow live networked virtual drumming between the FUNKarchitects studio at Bells Beach and the HLRS CAVE in Stuttgart. In this development, VDE's are set up on both locations and MIDI data is transmitted through a network from one VDE to another (Figure 9). This development may enable new forms of collaborative intercontinental dyadic augmented musico-spatial improvisation performance where drummers 'play off' each-others musical and spatial output.
The second extension involves the introduction of sound spatialisation to compliment the MIDIbased developments previously undertaken. Sound is live recorded from a USB microphone and sound wave data is extracted and, using a Fast Fourier Transformation (FFT), frequency data is extracted. These two additional live data recordings form the basis for a development of the Covise VDE code. We imagined these two data streams forming the upper and lower boundaries of an enclosing oval Sound Cave within the CAVE, with time providing the 3D depth of the projection in virtual space (Figure 8 ).

The upper surface of the Sound Cave projects a spatialisation of the sound wave form whilst the lower surface is derived from frequency data. The colour spectrum and surface characteristics (wireframe or surface), along with the virtual dimensions (heights of peaks and valleys, and depth of projection) of these two boundary surfaces can be adjusted in real time. Working within this Sound Cave, MIDI data is projected forward as polyrhythmic molecules. In this development, a recorded sound file can be used as the basis of the Sound Cave spatialisation, with the drummer improvising over these sounds to produce an independent PM spatialisation. These spatialisations can be complimented by the introduction of spatial sound and Virtual Instrumentation to create a vast set of musico-spatial performance opportunities.

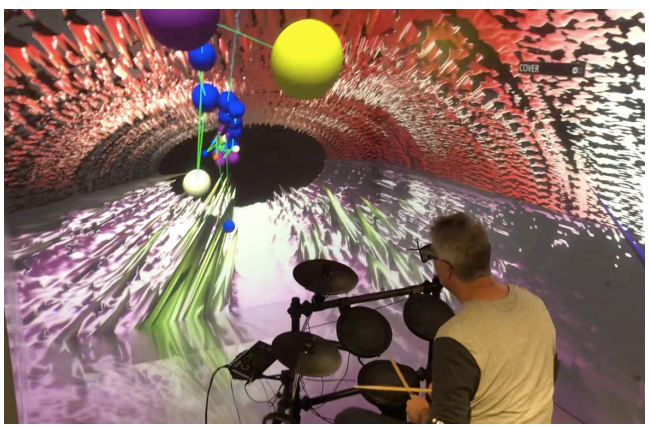




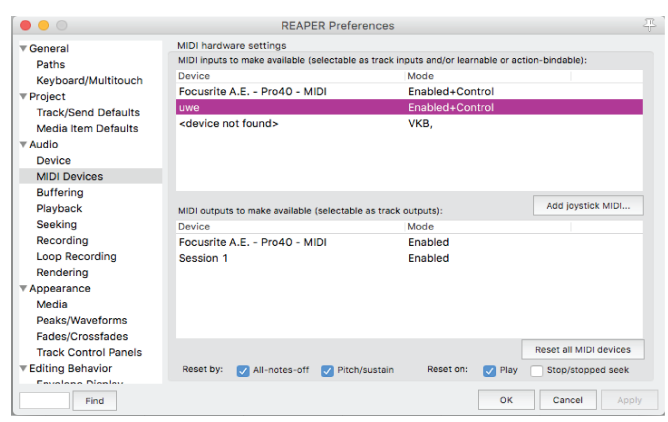

\section{CONCLUSIONS}

This research brings together musicians, architects and visualisation experts to develop the VDE platform. The VDE, as a means of transforming musical data into dynamic VR artefacts, provides a new spatial identity to polyrhtyhmic drumming. This spatial identity, whether dynamic (as event-time dynamics polyrhtyhmic molecules) or static (as PM virtual galleries), enhances affordances for understanding the complexities of drumming that are otherwise unavailable within the sonic domain.

In addition to being a useful tool for music information research, the VDE provides a rich platform for explorations of musical, spatial, musico-spatial and augmented musico-spatial improvisation. Through VI synthesised sounds, the VDE becomes a platform for both musical and spatial creativity. Extending the VDE into networked dyadic musico-spatial drumming and the Sound Cave effects multiple modalities for the extension of digital drumming beyond 'drumming's known worlds' (Bruford, 2015). Creative improvisation within the musical, spatial and musicospatial domains provides a methodology that informs iterative collaborative project development.

\section{REFERENCES}

Bruford, W 2015, Making it Work: Creative music performance and the Western kit drummer, Ph.D. Thesis, School of Arts, Faculty of Arts and Social Sciences, University of Surrey

Eckermann, J 1829, Conversations with Goethe in the Last

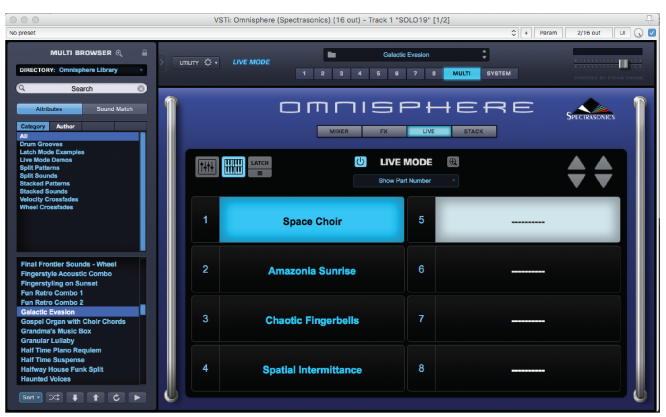

Figure 9

Networked VDE
Years of His Life, Hilliard, Gray, and Co, Boston, USA

Ham, J.J. 2017 'An architectural approach to 3D spatial notation', TENOR 2017: International Conference on Technologies for Music Notation and Representation, a Coruna, Spain

Ham, J.J. 2017a 'Improvisations in Polyrhythmic Spatiality', Back to the future: The next 50 years, 51st International Conference of the Architectural Science Association 2017, Wellington, NZ

Ham, J.J. 2018 'Exploring the intersection of music and architecture through spatial improvisation', CAADRIA 2018: Learning, Adapting and Prototyping Proceedings of the 23rd CAADRIA Conference, Beijing, China

Ham, J.J. 2018a, Improvising Polyrhythmic Space: Exploring a Continuum of Musico-Spatial Creative Practice, Ph.D. Thesis, RMIT University SIAL Sound Studios

J, Gibson 2009, The Theory of Affordances in the Ecological Approach to Visual Perception, Houghton Mifflin, Boston, USA

Maier, J.R. and Fadel, G.M. 2009, 'Affordance based design: A relational theory for design', Research in Engineering Design, 20(1), pp. 13-27

Martin, E (eds) 1994, Architecture as a translation of music, Princeton Architectural Press, New York, USA

Norman, D 2002, The Design of Everyday Things., Basic Books, New York, USA.

Novak, M 1994, 'Computation and Composition', in Martin, E (eds) 1994, Pamphlet Architecture: Architecture as a Translation of Music, Princeton Architectural Press, New York, USA. 